\title{
Carrier relaxation dynamics in an ultrafast all-optical modulator using an intersubband transition
}

$\operatorname{AUTHOR}(\mathrm{S})$ :

Asano, T; Yoshizawa, S; Noda, S

\section{CITATION:}

Asano, T... [et al]. Carrier relaxation dynamics in an ultrafast all-optical modulator using an intersubband transition. APPLIED PHYSICS LETTERS 2001, 79(27): 4509-4511

\section{ISSUE DATE:}

2001-12-31

URL:

http://hdl.handle.net/2433/50162

\section{RIGHT:}

Copyright 2001 American Institute of Physics. This article may be downloaded for personal use only. Any other use requires prior permission of the author and the American Institute of Physics. 


\title{
Carrier relaxation dynamics in an ultrafast all-optical modulator using an intersubband transition
}

\author{
T. Asano, ${ }^{\text {a) }}$ S. Yoshizawa, and S. Noda \\ Department of Electrical Science and Engineering, Kyoto University, Kyoto 606-8501, Japan
}

(Received 16 April 2001; accepted for publication 23 October 2001)

\begin{abstract}
An improvement of an all-optical modulation scheme that utilizes an intersubband transition is proposed and investigated in connection with intersubband carrier relaxation dynamics. $N$-doped GaAs/AlGaAs quantum wells are pumped by an ultrashort $(\sim 120 \mathrm{fs})$ intersubband-resonant pulse and probed by an interband-resonant white-light continuum. Ultrafast modulation speed of $\sim 0.4 \mathrm{ps}$ is demonstrated, which is faster than that of the previous scheme by as much as a factor of 10 . It is found that a large part of the carriers, which are excited from the first conduction subband to the second conduction subband, transfer to unknown trapping states at a relaxation time that is comparable to that of the pump-pulse duration. The existence of the carrier relaxation path via $L$ valley subbands is strongly suggested as the cause. (c) 2001 American Institute of Physics.
\end{abstract}

[DOI: $10.1063 / 1.1427154]$

An intersubband transition ${ }^{1}$ (ISBT) in a quantum well (QW) has attracted much interest due to unique characteristics such as an ultrafast carrier relaxation time. We have previously proposed an ultrafast all-optical modulation scheme that utilizes the ISBT [Fig. 1(a) $]^{2}$ that was aimed for use in ultrafast optical communication systems. The scheme utilizes an $n$-doped QW that contains at least two conduction subbands (CB1 and CB2) and one heavy-hole subband (HH1). An intense ISB-resonant light pulse (control light) can decrease the electron population in CB1 to modulate the absorption of $\mathrm{HH} 1-\mathrm{CB} 1$ interband resonant light (signal light). The recovery time of the modulation is determined by the carrier relaxation time of the ISBT. We have demonstrated modulation in GaAs/AlGaAs QWs, and have shown an ultrafast recovery time of $\sim 1$ ps [full width at half maximum (FWHM) ${ }^{3}$ However, the result also contained a slowly tailing component $(3-5 \mathrm{ps})$, which restricts the repetition rate of the modulation.

Understanding of the carrier relaxation dynamics is key to improving the modulation characteristics. The ISB relaxation mechanism in III-V semiconductor QWs has been studied experimentally ${ }^{4-13}$ and theoretically. ${ }^{14-18}$ Although the mechanism is not yet completely clear, it is basically considered to consist of two serial processes: ${ }^{4}$ (A) intersubband scattering from $\mathrm{CB} 2$ to $\mathrm{CB} 1$ via electron-longitudinaloptical (LO)-phonon scattering and (B) intrasubband relaxation within $\mathrm{CB} 1$ via electron-electron $(e-e)$ and electronphonon scattering. With regard to process $(\mathrm{A})$, the scattering time is no longer than $\sim 1$ ps in GaAs/AlGaAs QWs. ${ }^{3,18}$ For process $(B)$, there are some experimental reports ${ }^{9,11}$ that indicate that the intrasubband relaxation time is on the order of picoseconds. In addition, there are some reports that indicate the existence of a trap-like relaxation process $(\mathrm{C})^{7,8}$ in parallel with the process (A). By considering these factors, we have proposed an improved modulation scheme in which the signal light is tuned to the HH2-CB2 transition [Fig. 1(b)]. In the improved scheme, the effects of $(\mathrm{B})$ and $(\mathrm{C})$ can be

${ }^{a)}$ Electronic mail: tasano@kuee.kyoto-u.ac.jp avoided since the modulation speed is determined solely by the carrier lifetime in CB2.

In this letter, we investigate previous and improved modulation schemes simultaneously using white-light pump-probe method. The carrier relaxation dynamics within the conduction band are reconstructed from modulation profiles using a quantitative method. The relaxation mechanism is discussed and supported by numerical calculations.

The sample investigated was a multiple QW that consisted of 150 periods of $\mathrm{GaAs}(59 \AA) / \mathrm{Al}_{0.35} \mathrm{Ga}_{0.65} \mathrm{As}(150 \AA)$. Only the barriers were selectively doped with $\mathrm{Si}$ (1.5 $\times 10^{18} \mathrm{~cm}^{-3}$ ), where the Fermi level is calculated to lie about $70 \mathrm{meV}$ above the bottom of CB1. The energy separations between CB1 and CB2, HH1 (top) and CB1 (bottom) and $\mathrm{HH} 2$ (top) and $\mathrm{CB} 2$ (bottom), were determined to be $0.172,1.50$ and $1.72 \mathrm{eV}$, respectively, by conventional absorption measurements [inset of Fig. 2(b)]. In modulation experiments, the sample was pumped by an ISB-resonant light pulse $(0.17 \mathrm{eV}, \sim 120 \mathrm{fs})$ and was probed by a whitelight continuum that ranged from 1.45 to $1.90 \mathrm{eV}$. Optical parametric amplification and differential frequency mixing techniques were used to generate the pump pulse, and a water cell was utilized to generate the white-light continuum. The pump and probe pulses were incident onto the sample, the substrate of which was removed by selective etching, at

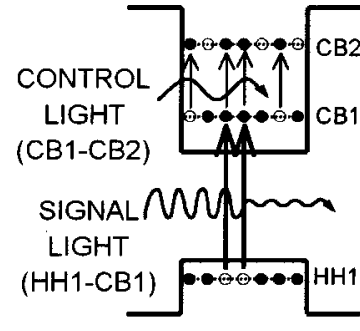

(a) Previous

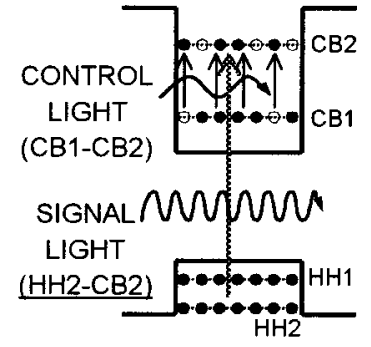

(b) Improved
FIG. 1. Schematic diagrams of all-optical modulation using an intersubband transition in $n$-doped quantum wells: (a) previously proposed scheme, which utilizes the carrier dynamics in the first conduction subband, and (b) improved scheme, which utilizes the carrier dynamics in the second conduction subband. 
(a)
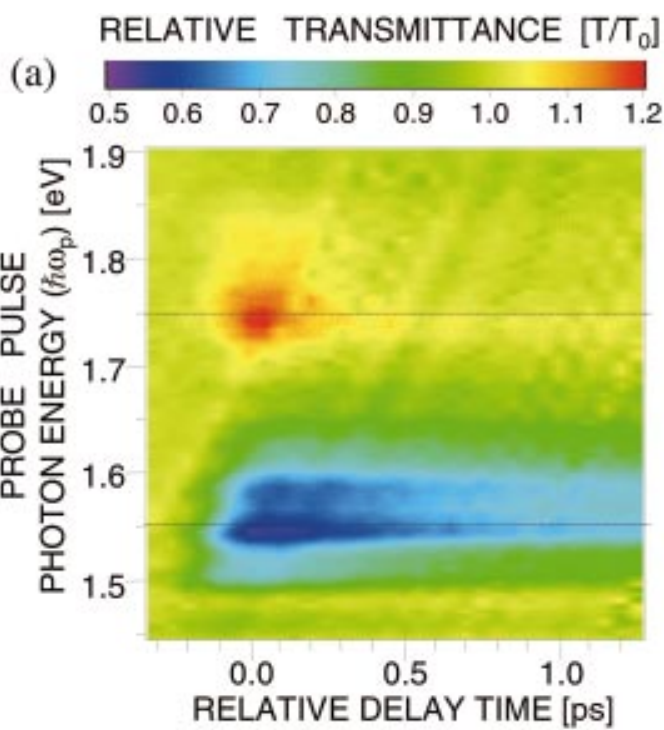

(b)

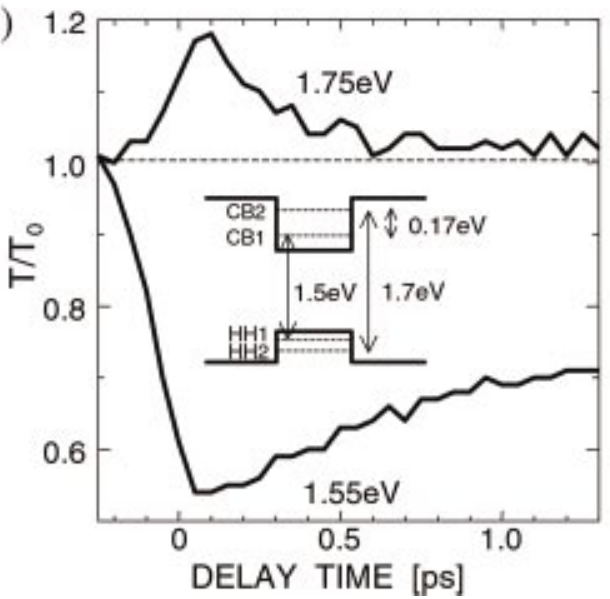

FIG. 2. (Color) Example of an intersubband pump and interband white-light probe experiment: (a) three-dimensional view and (b) a cross section at 1.55 $\mathrm{eV}$ (lower line) and a cross section at $1.75 \mathrm{eV}$ (upper line). Inset: Energy separations between subbands measured by ordinary absorption measurements.

nearly the Brewster angle with $p$ polarization. All the measurements were carried out at room temperature.

Figure 2(a) shows one example of the modulation profile $\left(T / T_{0}\right)$, where the effective pump-pulse energy density was about $100 \mathrm{fJ} / \mu \mathrm{m}^{2}$. The increase of $T / T_{0}$ in a photon energy range of $1.5-1.7 \mathrm{eV}$ reflects the modulation in the $\mathrm{HH} 1-$ CB1 transition (the previous scheme). The cross section at $1.55 \mathrm{eV}$ shows double exponential relaxation [Fig. 2(b)], and the fast and slow components of the relaxation times are about 2.5 and $6 \mathrm{ps}$, respectively. The increase of $T / T_{0}$ in a photon energy range of $1.7-1.9 \mathrm{eV}$ reflects the modulation in the HH2-CB2 transition (the improved scheme). The cross section at $1.75 \mathrm{eV}$ shows single exponential relaxation [Fig. 2 (b)], and the relaxation time is about $\sim 0.4$ ps. The overall response time of the improved scheme was faster than that of the previous scheme by a factor of almost 10 . This is partly due to the avoidance of the intrasubband relaxation process, as we expected. However, the observed relaxation time $(\sim 0.4 \mathrm{ps})$ is much shorter than that expected from LOphonon scattering theory $(\sim 1 \mathrm{ps}) .{ }^{14-18}$

In order to analyze the relaxation mechanism, we reconDownloaded 04 Mar 2008 to 130.54.110.22 Redistribution subject

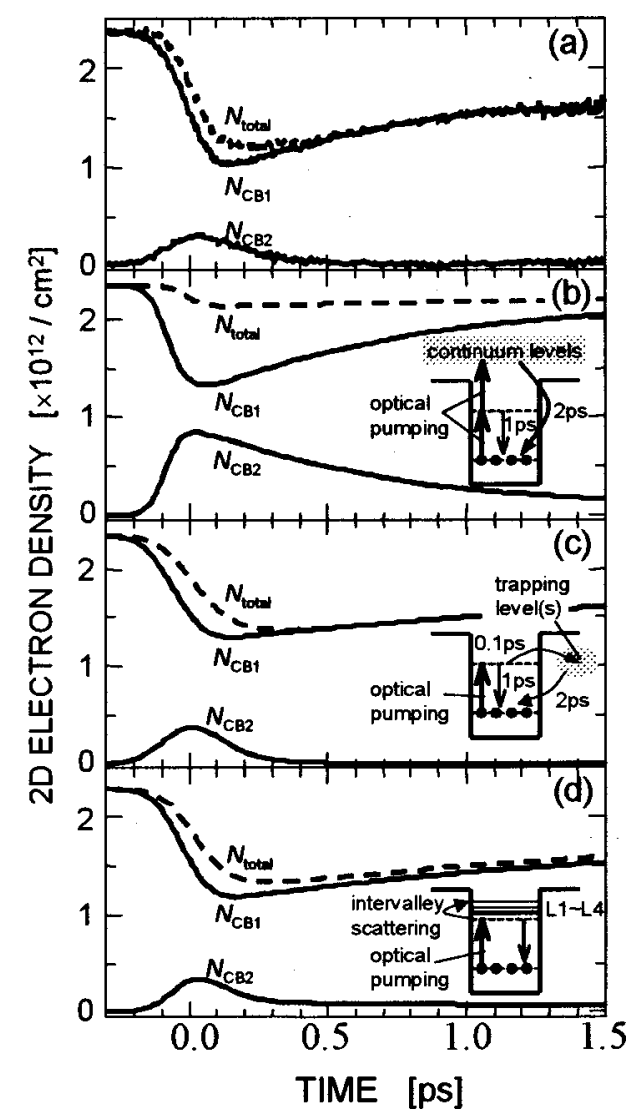

FIG. 3. Time profile of the total electron population within each subband $\left(N_{\mathrm{CB} 1}\right.$ and $\left.N_{\mathrm{CB} 2}\right)$ and their sum $\left(N_{\text {total }}\right)$. (a) Experimental data converted from the measurement result, (b) rate equation analysis assuming optical pumping from $\mathrm{CB} 2$ to continuum levels, (c) rate equation analysis assuming scattering from $\mathrm{CB} 2$ to the trapping state with a phenomenological relaxation time of $0.1 \mathrm{ps}$, and (d) Monte Carlo analysis that considers the four lowest subbands in the $L$ valley minimum.

structed the electron distributions within the conduction subbands from the modulation profile. This was carried out by a semi-empirical method that utilizes theoretical fitting of the linear interband absorption spectrum. Next, we integrated the electron distribution along the energy axis, and obtained a time profile of the total electron populations in CB1 and CB2 (denoted as $N_{\mathrm{CB} 1}$ and $N_{\mathrm{CB} 2}$, respectively). The profile reflects the intersubband electron relaxation only, and does not reflect the intrasubband carrier relaxation. Figure 3(a) shows the carrier relaxation profile obtained from the data shown in Fig. 2. We can see that the increase of $N_{\mathrm{CB} 2}$ is distinctly smaller than the decrease of $N_{\mathrm{CB} 1}$. About $1.3 \times 10^{16} \mathrm{~m}^{-2}$ electrons (almost half of the doped electrons) are removed from CB1 at the peak, but only about $25 \%$ of them are observed in CB2. It is thought that a large part of the electrons excited to CB2 instantly transfer (in a time comparable to the pump-pulse duration) to unknown trapping level(s) where the electron population does not influence the transmittance of the probe light. The trapped electrons are considered to relax to CB1 with a slow relaxation time on the order of picoseconds. A relaxation process other than direct CB2CB1 scattering should be considered to explain the results.

At first, we considered a hypothesis that the electrons excited to CB2 are again excited to continuum levels that exist over the top of the barrier layer by the same pump pulse [inset of Fig. 3(b)]. The absorption cross section of the CB2 AIP license or copyright; see http://apl.aip.org/apl/copyright.jsp 
continuum level transition is calculated to be almost $40 \%$ that of the $\mathrm{CB} 1-\mathrm{CB} 2$ transition. We have carried out a rate equation calculation of the carrier relaxation process by assuming that the electrons that are excited to continuum levels relax directly to $\mathrm{CB} 1$ with a phenomenological relaxation time of $2 \mathrm{ps}$. The result obtained did not agree with that of the experiment as can be seen in Fig. 3(b). Only small parts of the electrons excited to CB2 are again excited to the continuum levels, which indicates that the mechanism plays a minor role in the relaxation process.

Next, we investigated the hypothesis that excited electrons transfer to a trapping level(s) that is located energetically near CB2 by means of some scattering mechanisms. The rate equation analysis [Fig. 3(c)] showed good agreement with the experiment [Fig. 3(a)] when we assumed a phenomenological scattering time of $\sim 0.1$ ps for electron transfer from CB2 to the trapping level(s). In order to clarify details of the mechanism, we investigated the following two models for the trapping states.

(1) Barrier subbands: ${ }^{8}$ Since barrier-doped QWs are utilized in the experiment, subbands are also formed near the bottom of the potential bend in the barrier. The lowest barrier subband is formed in the $\Gamma$ valley and is calculated to lie about $35 \mathrm{meV}$ above CB2.

(2) $L$ (Ref. 7) and $X$ valley subbands: Subbands formed in the $L(X)$ valley, which are spatially confined in the GaAs (AlGaAs) layer, can exist energetically near CB2. They are calculated to lie about $50 \mathrm{meV}$ above CB2.

We verified model (1) by carrying out the same experiment on the newly fabricated QWs, where only the well layers were doped to prohibit the formation of barrier subbands. However, the electron relaxation profiles obtained were almost same as those obtained in the barrier-doped QWs. Model (1) was shown to play a minor role in the relaxation process. For verification of model (2), the scattering times were calculated using the intervalley deformation potential model $^{19}$ and the parameters reported in Ref. 20. The calculated scattering time from CB2 to the first $L$ valley subband (denoted a $L 1)$ was about 0.3 ps $(0.1 \mathrm{ps})$ for the phonon absorption (emission) process. (Even if L1 lies 50 meV above $C B 2$, both processes are possible since the average momentum energy of electrons is enough large. The Fermi level lies 70 meV above the CB1 bottom before excitation.) Backscattering from the $L$ to $\Gamma$ subbands is slower than the forward scattering by a factor of more than 10 due to the difference in density of the final states. For $X$ valleys, the scattering time from $\mathrm{CB} 2$ to the first $X$ valley subband is calculated to be on the order of picoseconds, and is due to spatial separation of the wave functions. The results for $\Gamma-L$ scattering are consistent with the experiments but those for $\Gamma-X$ scattering are not.

In order to demonstrate the $\Gamma-L$ scattering model, we have carried out an ensemble Monte Carlo simulation of the carrier relaxation process. ${ }^{21-23}$ Here, we took into account the intersubband LO-phonon, intrasubband LO-phonon, intrasubband $e-e$, and intervalley $(\Gamma-L)$ deformation potential scattering. Phonon confinement effects, hot phonon effects, and dynamical Coulomb screening effects were considered. With regard to $\Gamma-L$ scattering, we took into ac- count the four lowest $L$ subbands. The $\Gamma-L$ scattering by the $e-e$ process was ignored since a large change in momentum is required. We compared the theoretical and the experimental results by changing the energy separation between CB2 and $L 1$. As a result, it was found that good agreement with the experiment is obtained when $L 1$ is assumed to lie 25 meV above CB2 [Fig. 3(d)]. Although the energy separation assumed $(25 \mathrm{meV})$ is smaller than that estimated before $(50$ $\mathrm{meV}$ ), we think that the relatively small discrepancy can be explained by an error in the estimation. (The energy separation is evaluated from the quantization energies of the $L 1$ and CB2 subbands and the energy separation between the $\Gamma$ and $L$ minima. ${ }^{20}$ ) The agreement between Figs. 3(a) and 3(d) clearly demonstrates the importance of the $\Gamma-L$ scattering process. A small discrepancy still remains that is considered to be due to the other minor relaxation process ignored here, for example, optical pumping from CB2 to continuum levels.

In summary, we have proposed an improved modulation scheme that utilizes the carrier dynamics in the second conduction subband. Ultrafast modulation response time of $\sim 0.4$ ps has been demonstrated using the GaAs/AlGaAs QWs. It is strongly suggested that the intervalley deformation potential scattering between the $L$ and $\Gamma$ subbands plays an important role in the carrier relaxation mechanism, in addition to usual intersubband LO-phonon scattering.

This work was supported in part by a Grant-in-Aid for Scientific Research from the Ministry of Education, Culture, Sports Science, and Technology of Japan, the Femtosecond Technology Research Association, which is supported by New Energy and Industrial Technology Development Organization, and Venture Business Laboratory, Kyoto University.

${ }^{1}$ L. C. West and S. J. Eglash, Appl. Phys. Lett. 46, 1156 (1985).

${ }^{2}$ S. Noda, T. Uemura, T. Yamashita, and A. Sasaki, J. Appl. Phys. 68, 6529 (1990).

${ }^{3}$ T. Asano, S. Yoshizawa, and S. Noda, Appl. Phys. Lett. 77, 19 (2000).

${ }^{4}$ A. Seilmeier, H.-J. Hübner, G. Abstreiter, G. Weimann, and W. Schlapp, Phys. Rev. Lett. 59, 1345 (1987).

${ }^{5}$ D. Y. Oberli, D. R. Wake, M. V. Klein, J. Klem, T. Henderson, and H. Morkoç, Phys. Rev. Lett. 59, 696 (1987).

${ }^{6}$ J. A. Levenson, G. Dolique, J. L. Oudar, and I. Abram, Phys. Rev. B 41, 3688 (1990)

${ }^{7}$ J. L. Educato, L. P. Leburton, J. Wang, and D. W. Bailey, Phys. Rev. B 44, 8365 (1991).

${ }^{8}$ J. Baier, I. M. Bayanov, U. Plödereder, and A. Seilmeier, Superlattices Microstruct. 19, 9 (1996).

${ }^{9}$ S. Lutgen, R. A. Kaindl, M. Woerner, T. Elsaesser, A. Hase, H. Kunzel, M. Gulia, D. Meglio, and P. Lugli, Phys. Rev. Lett. 77, 3657 (1996).

${ }^{10}$ T. A. Gardiner, J. V. Vandyshev, G. W. Wicks, and P. M. Fauchet, Abstracts of QELS'97, 1997, p. 137.

${ }^{11}$ R. Duer, I. Shtrichman, D. Gershoni, and E. Ehrenfreund, Phys. Rev. B 78, 3919 (1997)

${ }^{12}$ T. Asano, S. Noda, and K. Tomoda, Appl. Phys. Lett. 74, 1418 (1999).

${ }^{13}$ N. Iizuka, K. Kaneko, N. Suzuki, T. Asano, S. Noda, and O. Wada, Appl. Phys. Lett. 77, 648 (2000).

${ }^{14}$ R. Lassing, Phys. Rev. B 30, 7132 (1984).

${ }^{15}$ K. Huang and B. Zhu, Phys. Rev. B 38, 13377 (1988).

${ }^{16}$ B. K. Ridley, Phys. Rev. B 39, 5282 (1989).

${ }^{17}$ S. Rudin and T. L. Reinecke, Phys. Rev. B 41, 7713 (1990).

${ }^{18}$ H. Rücker, E. Molinari, and P. Lugli, Phys. Rev. B 45, 6747 (1992).

${ }^{19}$ H. Tanimoto, N. Yasuda, K. Taniguchi, and C. Hamaguchi, Jpn. J. Appl. Phys., Part 1 27, 563 (1988).

${ }^{20}$ M. A. Littlejohn, J. R. Hauser, and T. H. Glisson, J. Appl. Phys. 48, 4587 (1977).

${ }^{21}$ W. Fawcett, A. D. Bordman, and S. Swain, J. Phys. Chem. Solids 31, 1963 (1970).

${ }^{22}$ M. A. Osman and D. K Ferry, Phys. Rev. B 36, 6018 (1987).

${ }^{23}$ S. M. Goodnick and P. Lugli, Phys. Rev. B 37, 2578 (1988). 